Präv Gesundheitsf 2022 $17: 83$ https://doi.org/10.1007/s11553-021-00902-4 Online publiziert: 8 . September 2021 (c) Der/die Autor(en) 2021

Max Weniger ${ }^{1,2} \cdot$ Katja Beesdo-Baum $^{1,2} \cdot$ Veit Roessner $^{3} \cdot$ Helene Hense $^{4} \cdot$ Susanne Knappe' ${ }^{1}$ (D)

'Institut für Klinische Psychologie und Psychotherapie, Technische Universität Dresden, Dresden, Deutschland; ' ${ }^{2}$ Institut für Klinische Psychologie und Psychotherapie, Behaviorale Epidemiologie, Technische Universität Dresden, Dresden, Deutschland; ${ }^{3}$ Klinik und Poliklinik für Kinder- und Jugendpsychiatrie und -psychotherapie, Universitätsklinikum und Medizinische Fakultät Carl Gustav Carus, Technische Universität Dresden, Dresden, Deutschland; ${ }^{4}$ Forschungsverbund Public Health Sachsen, Zentrum für Evidenzbasierte Gesundheitsversorgung (ZEGV), Universitätsklinikum und Medizinische Fakultät Carl Gustav Carus, Technische Universität Dresden, Dresden, Deutschland

\title{
Erratum zu: Wie gelingt die Prävention psychischer Beschwerden?
}

\section{Von der Vorsorgeuntersuchung zur indikativen Präventionsmaßnahme bei emotionalen und Verhaltensauffälligkeiten im Vor- und Grundschulalter: eine prospektive Implementationsstudie}

\section{Erratum zu:}

Präv Gesundheitsf 2021

https://doi.org/10.1007/s11553-021-

00838-9

In der ursprünglichen Version des Beitrags war leider ein Fehler bei einer Prävalenzangabe enthalten. Deshalb wurde im Abschnitt „Hintergrund und Fragestellung“ der erste Satz geändert in „Unter den 3-bis 6-jährigen Kindern leiden 6,6\% bzw. 15,8\% und unter den 7- bis 10-Jährigen $10,4 \%$ bzw. $15,1 \%$ an emotionalen oder Verhaltensauffälligkeiten [14, 21].“

Außerdem wurde im zweiten Satz im Abschnitt „Stichprobe“ die Referenzangabe von [10] zu [14] korrigiert.

Der Originalbeitrag wurde korrigiert.

\section{Korrespondenzadresse}

\section{Max Wenige}

Institut für Klinische Psychologie und

Psychotherapie, Technische Universität

Dresden

Dresden, Deutschland

Max.Weniger@tu-dresden.de
Open Access. Dieser Artikel wird unter der Creative Commons Namensnennung 4.0 International Lizenz veröffentlicht, welche die Nutzung, Vervielfältigung, Bearbeitung, Verbreitung und Wiedergabe in jeglichem Medium und Format erlaubt, sofern Sie den/die ursprünglichen Autor(en) und die Quelle ordnungsgemäß nennen, einen Link zur Creative Commons Lizenz beifügen und angeben, ob Änderungen vorgenommen wurden.

Die in diesem Artikel enthaltenen Bilder und sonstiges Drittmaterial unterliegen ebenfalls der genannten Creative Commons Lizenz, sofern sich aus der Abbildungslegende nichts anderes ergibt. Sofern das betreffende Material nicht unter der genannten Creative Commons Lizenz steht und die betreffende Handlung nicht nach gesetzlichen Vorschriften erlaubt ist, ist für die oben aufgeführten Weiterverwendungen des Materials die Einwilligung des jeweiligen Rechteinhabers einzuholen.

Weitere Details zur Lizenz entnehmen Sie bitte der Lizenzinformation auf http://creativecommons.org/ licenses/by/4.0/deed.de.

Die Online-Version des Originalartikels ist unter https://doi.org/10.1007/s11553-021-00838-9 zu finden. 